\title{
SCOLIOSIS ACCOMPANYING CHRONIC INFECTED OPEN PNEUMOTHORAX: ITS CAUSATION AND CORRECTION*
}

\author{
FRASER B. GURD, B.A., M.D. \\ Assistant Surgeon to the Montreal General Hospital \\ MONTREAL, CANADA
}

Long-continued suppuration of large cavities in open pneumothorax is followed by a deviation of the spine to the healthy side. During the last two years, I have had an unusual opportunity to study this type of case. ${ }^{1}$ As a result of these observations, I believe it has been demonstrated that the cause of the scoliosis in these cases is pressure, due to the ribs on the diseased side, proof of which is afforded by the effect of extensive thoracotomy in correcting the deformity.

When the lung is firmly held in a collapsed state by the dense enveloping membrane, which develops in long standing cases of large empyema cavities, nature attempts to obliterate the dead space by approximating the chest wall to the fixed lung. The anterior extremities of the ribs fall, making their horizontal direction more vertical; the intercostal spaces become obliterated, and in very long standing cases, such as the case described herewith, the ribs overlap one another. The shoulder on the affected side drops from 2 to $4 \mathrm{~cm}$., or more, and the middorsal and the lower dorsal spine are pushed over to the unaffected side. In this way a well marked scoliosis, with its concavity toward the affected sicle of the chest, is produced. The deviation of the spinous processes from the midline may be $3 \mathrm{~cm}$., or more.

In performing decortication, or pneumolysis, in a series of longstanding cases, I have found it necessary to employ, in several cases, a very extensive form of thoracotomy, in order to obtain an adequate exposure of the thorax. As a concomitant of the favorable results of this operation, it has been noted that the scoliosis, which was present in these cases prior to operation, is either entirely corrected or much improved.

If a simple intercostal thoracotomy is performed, normal alinement of the spine is dependent on return of the affected half of the chest to full function. This, at best, must be slow, and it is difficult to imagine its possibility in view of the extreme thickness ( 1 to $2 \mathrm{~cm}$., or more) of the parietal pleura, and the intercostal fibrosis present in all the more chronic cases.

* Read before the fourth annual meeting of the American Association for Thoracic Surgery, Boston, June 6, 1921.

1. Gurd, F. B.: Canad. M. A. J. 2:408 (June) 1921. 
When, on the other hand, a portion of the lower part of the chest wall is removed, and the upper ribs are divided, there is evident at once a marked change in the shape of the chest. The spine, free from the pressure of the ribs, straightens, and the shoulder returns to its proper position. Both the anteroposterior and the transverse diameters of the diseased half of the chest remain less than those of the healthy side; but the disfiguring deformity is overcome.

In performing this operation, the incision follows the line of the ninth rib from the midline or anterior axillary line, backward to the

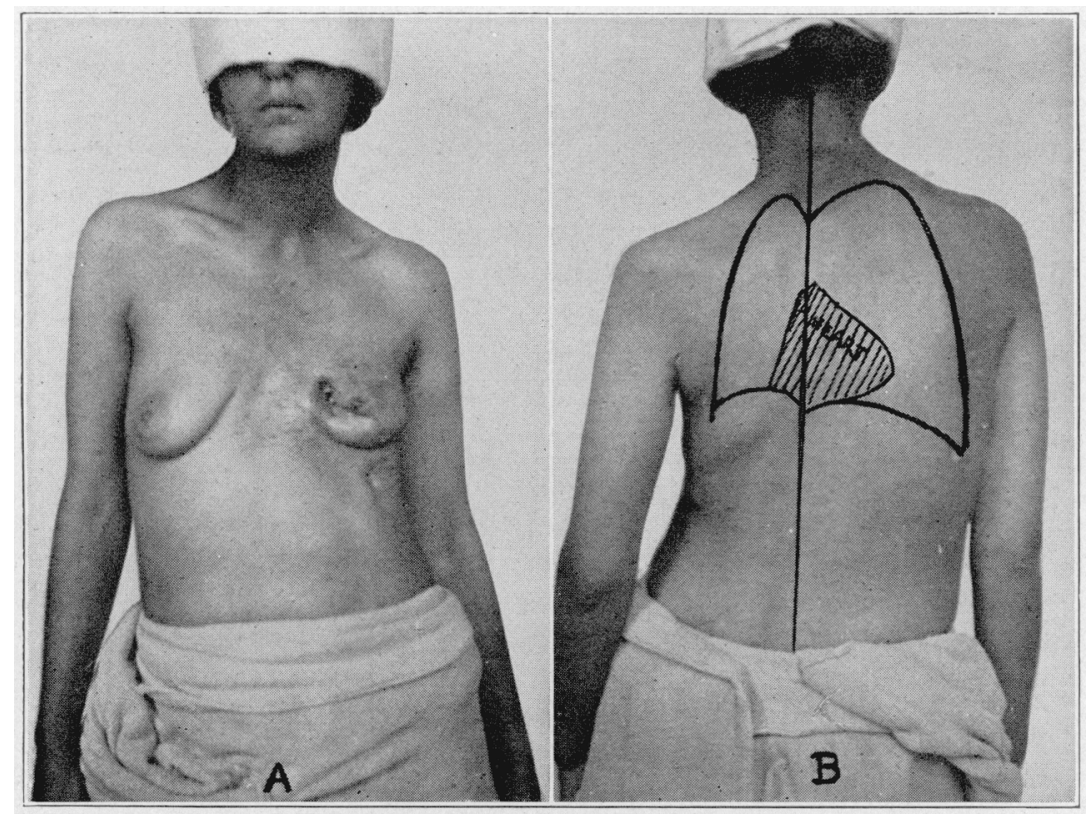

Tig. 1.-A, marked deformity and discharging sinus through the left breast in patient B. C.; 3 , diagrammatic drawing on photograph. showing marked reduction in size of left half of chest, together with lateral curvature of the spine.

angle of the scapula, where it curves upward to about the level of the spine of the scapula and ends midway between the scapula and the spinous processes. From 10 to $15 \mathrm{~cm}$. of the eighth and ninth, and occasionally of the tenth rib, are removed, together with the intervening intercostal tissues, and the underlying thickened parietal pleura. The removal of this portion of the wall permits manual, and, to a limited extent, visual exploration of the cavity, and provides for the introduction of tubes for the subsequent use of discontinuous irrigation with surgical solution of chlorinated soda (Dakin's solution). As a general rule, further operative interference should not be undertaken at the primary operation. 


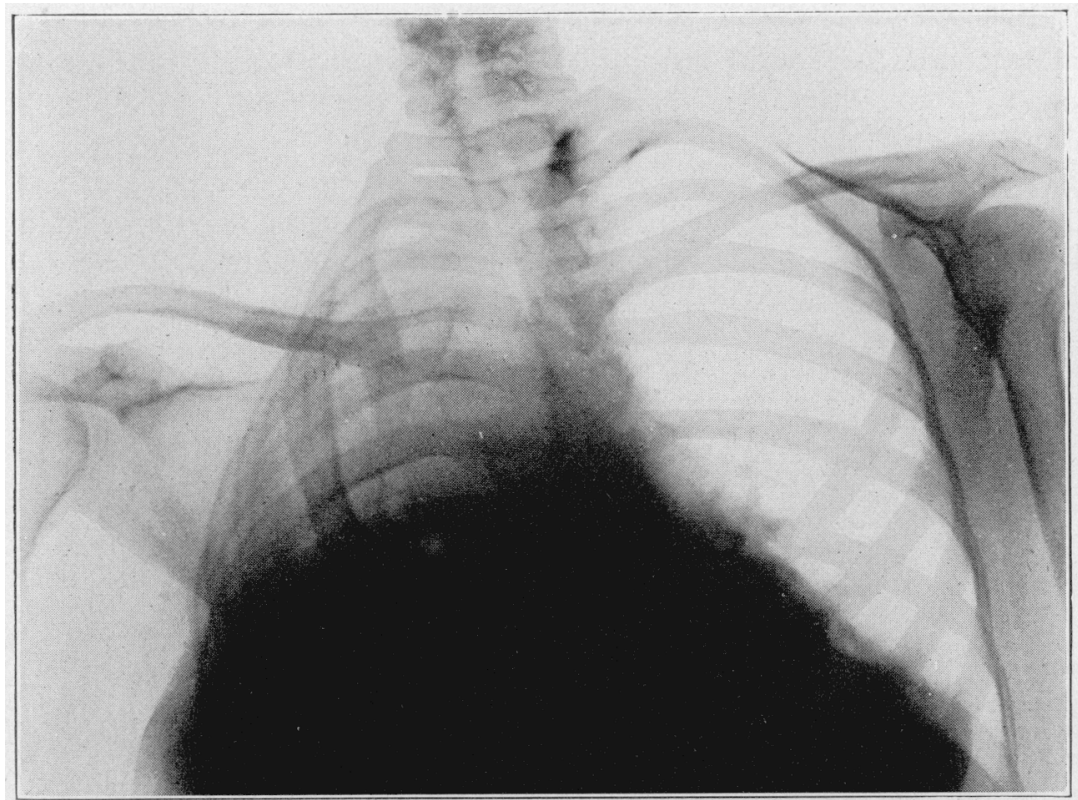

Fig. 2.-Roentgenogram (B. C.), taken before operation, showing marked curvature of the spine and displacement of the heart to the right.

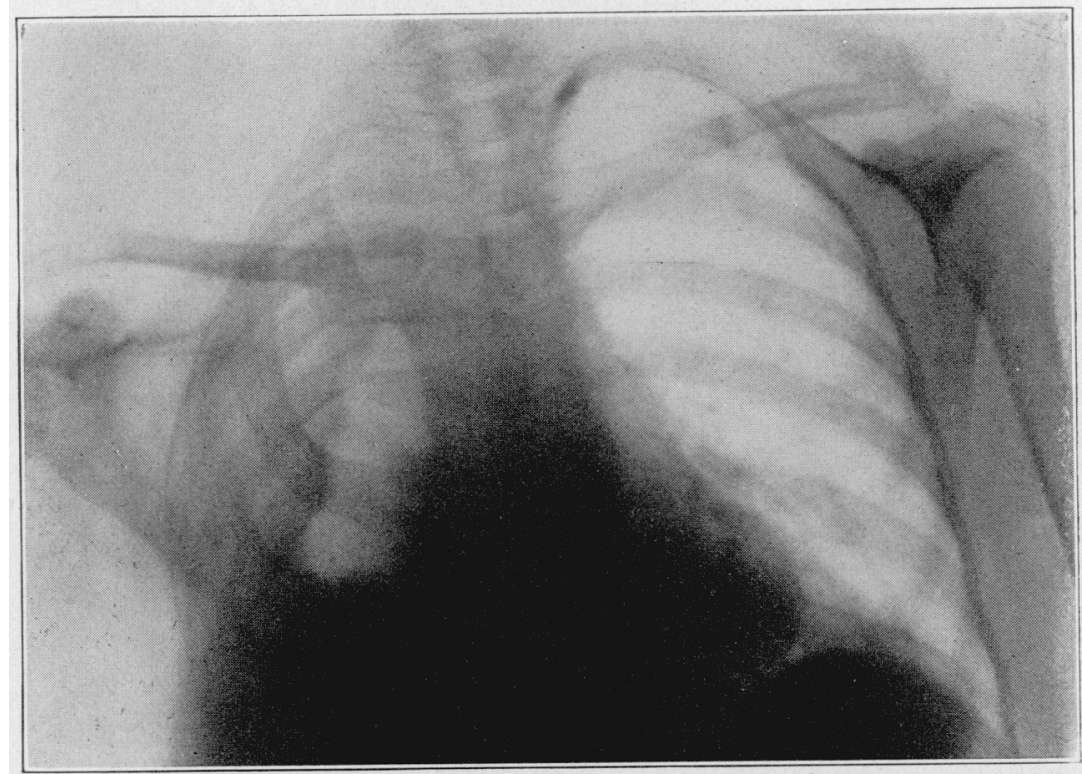

Fig. 3--Roentgenogram taken one week after operation showing partial correction of deformity; the clear area on the left side of the chest is the site of removal of thickened parietal pleura. 
Following this partial thoracotomy, discontinuous irrigation of the avity should be carried out for two or three weeks, then the second stage of the operation should be undertaken. At the second operaticn, 5 or $7 \mathrm{~cm}$. of the seventh rib is excised, and the sixth, fifth, fourth, and, occasionally, the third, ribs are sectioned. I have not found it necessary or desirable to cut the first two ribs, although that would probably correct more completely the lateral curvature. Such an exposure permits satisfactory intrathoracic operation in the most clensely walled chests, and is followed by straightening of the spine.

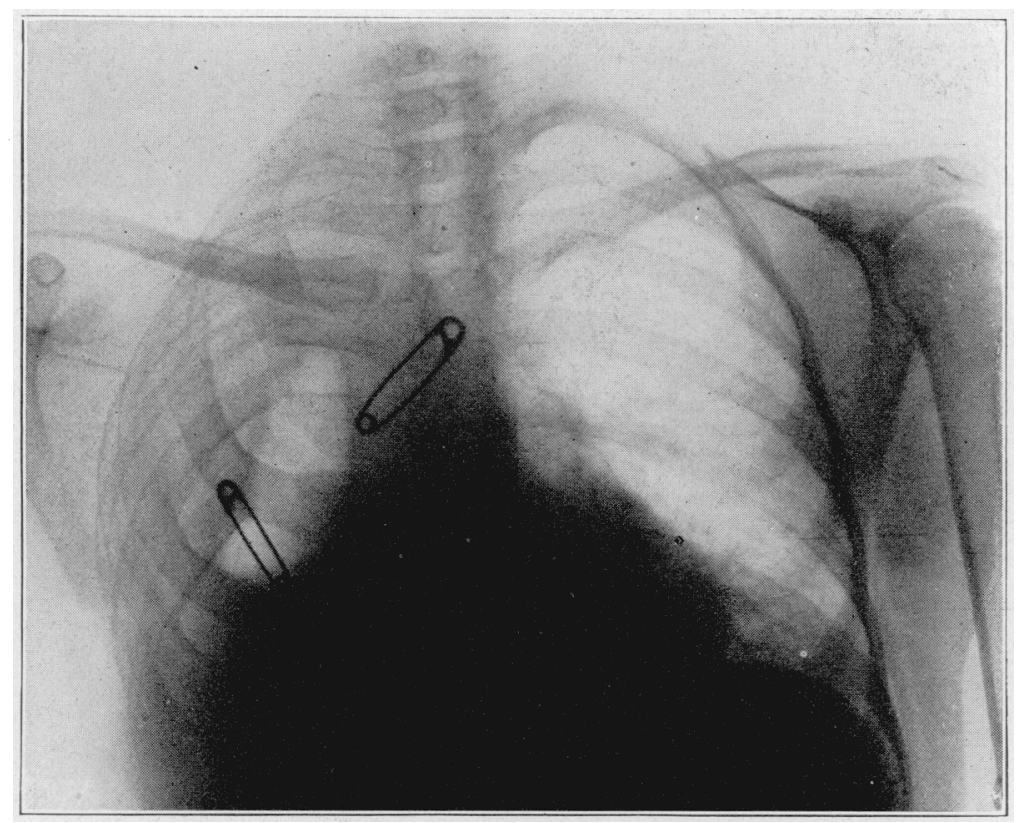

Fig. 4.-Roentgenogram (B. C.), taken one month after operation, showing increase in the size of the left half of the chest, as well as almost complete correction of the deformity.

I have usually employed paravertebral procain anesthesia. assisted from time to time during the operation by light chloroform anesthesia.

The case reported herewith exemplifies the usefulness of decortication in obliterating the open pneumothorax, arresting the suppuration, and correcting the scoliosis.

\section{REPC:RT OF CASE}

I/i:tory-B. C., woman, aged 23, was admitted to the Montreal General Hospital, Sept. 27, 1920. Since May, 1916, she had had a left sided empyema which upon three occasions had ruptured spontaneously. Two sinuses in the lower part of the chest were healed at the time of admission; but a moderate purulent clischarge poured from an opening through the left breast situated above and toward the midline from the nipple. She had lost 55 pounds $(25 \mathrm{~kg}$.) and was very emaciated and weak, although able to walk about. 


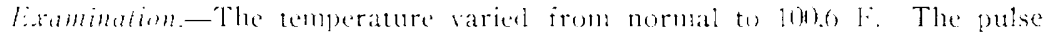

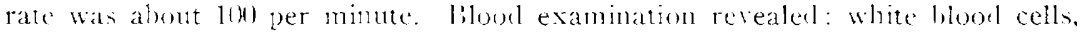

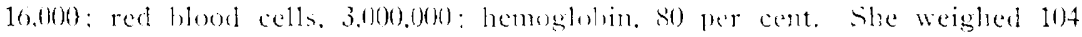
pounds (47 k. . .).

The hare was displated completely wo the right sile of the chest the apex heat being palpable beiow the right nipple. Plysical and ronentgenologic examination indicated a complete cavity of the left chest.

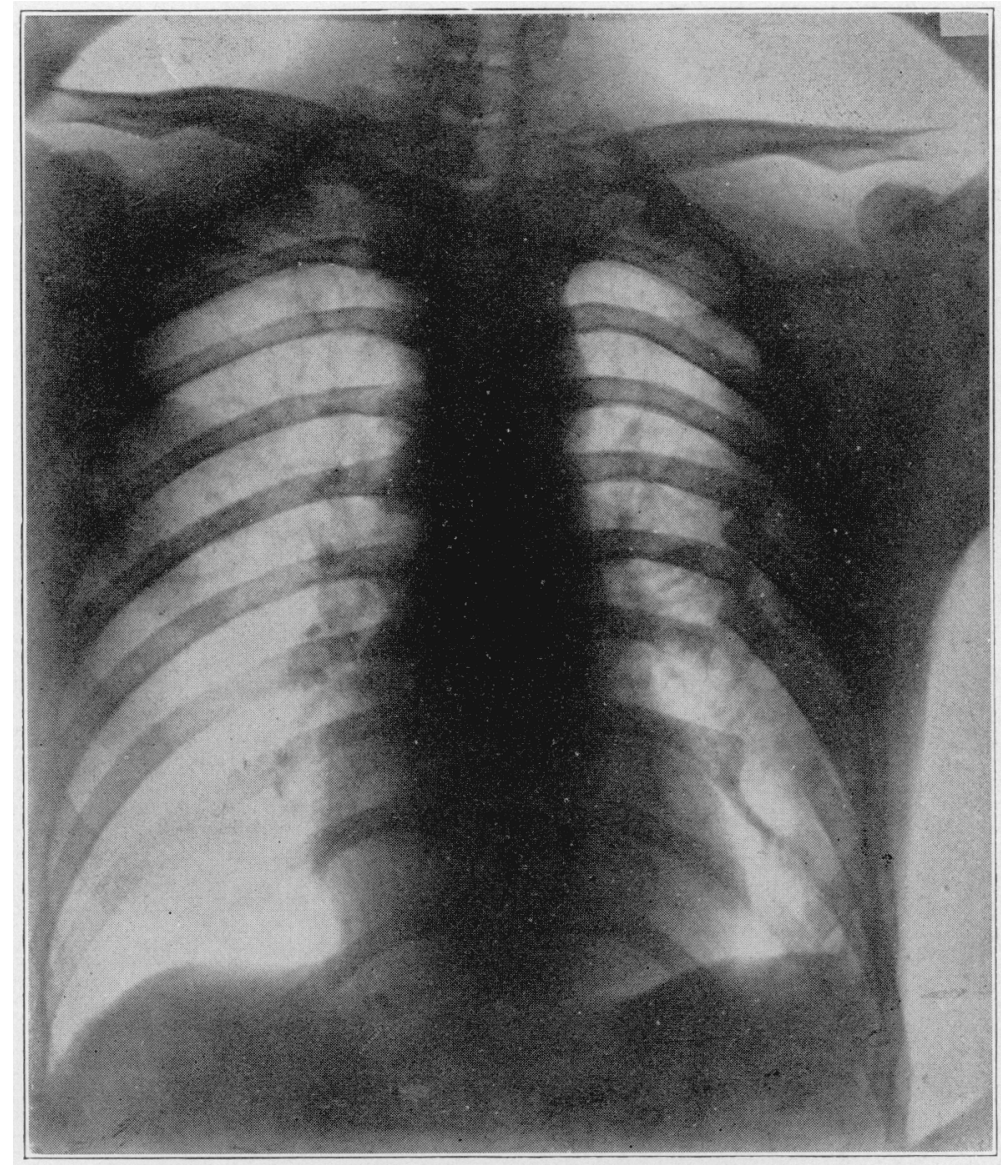

Fig. 5.-Roentgenogram (B. C.), taken one mouth after thoracotomy and pulmonary decortication, showing clearly the tendency of the ribs an the atfected sirle to werlap one another following section.

I marked deformity was present. The lefit sile of the diest was Hattened both in front and lehinel; the axilla was depressed; the shoukler droped, and there was a marked lateral deviation of the spine, with comsexity to the right. The right side of the chest appeared fuller than normal, and respiratory mosements were alsent on the left side. The spinsus procesies leviated to the right, between 5 and $5 \mathrm{~cm}$. from the midline. 
Operation.-September 30 , an incision, $15 \mathrm{~cm}$. long, was made over the ninth rib in the axillary line. The ribs overlapped one another by one-third of their width; $9 \mathrm{~cm}$. of the eighth, ninth, and tenth ribs were resected. The parietal pleura was extremely dense and hard, and was approximately $2 \mathrm{~cm}$. thick. The cavity, which contained about 40 c.c. of foul, thick pus, was freely exposed. There was no lung in the left side of the chest, and no respiratory or cardiac movements could be felt. Owing to the marked deformity of the chest, the whole left half of the thorax was small. A few adhesions crossing the cavity were broken down, and the surface cleaned with gauze. Two Carrel tubes were sutured in place, and the wound closed.

Course.-For two weeks following operation, discontinuous irrigation with Dakin's solution was carried out four times a day. During the irrigations, the patient was placed in such a position that as large an amount of the cavity was exposed to the solution as possible. For ten days, the discharge was foul. It then improved rapidly, and by the time of the second operation it was free from odor, although there was a moderate amount of purulent discharge on the dressings.

Second Operation.-Under intratracheal anesthesia, fifteen days after the preliminary operation, the incision made at the last operation was opened, and continued upward between the spinous processes and the scapula as far as the upper angle of the scapula. The scapular muscles and the remainder of the latissimus dorsi were cut transversely. Four ribs were sectioned upward from the medial border of the previous operation; $7 \mathrm{~cm}$. of the seventh ril was excised, and shorter portions of the fifth and sixth ribs. The thickened pleura was partly excised, to obtain satisfactory exposure, a procedure made necessary by intercostal fibrous tissue and thickened parietal pleura. With difficulty, the covering over the lungs was removed completely over the lower two thirds; above this point it was separated from the lung surface and incised. The covering over the pericardium, and over the greater portion of the diaphragm, was also removed. Considerable bubbling occurred from the surface of the lung over parts which had been torn during decortication. Although the patient could not be induced to cough, it was obvious that the lung was still expansile. The wound was closed by catgut sutures for the muscle, and silkworm-gint for the skin. Two long paraffin gauze bismuth-iodoform-petrolatum-paste drains were inserted in the apex of the cavity and brought out through the lower anterior portion of the wound.

Result.-The patient stood the operation well. For forty-eight hours she was moderately weak, although her pulse did not rise above 100 per minute, and the temperature remained practically normal. The drains, behind which was found about 20 c.c. of bloody serum, were removed forty-eight hours after operation. The wound was allowed to close, and forty-eight hours later a director was passed into the chest. Only a few drops of clear serum was evacuated. At no time, subsequently, was there the slightest discharge from the wound.

The fourth day following operation, the following note was made: The patient feels strong and is suffering but little discomtort. The deformity of the chest has been corrected to a considerable degree. Scoliosis is not more than $2.5 \mathrm{~cm}$. from the midline.

The patient was discharged from the hospital Nov. 4, 1920. The wound was then soundly healed; her general appearance was much improved, and she was 


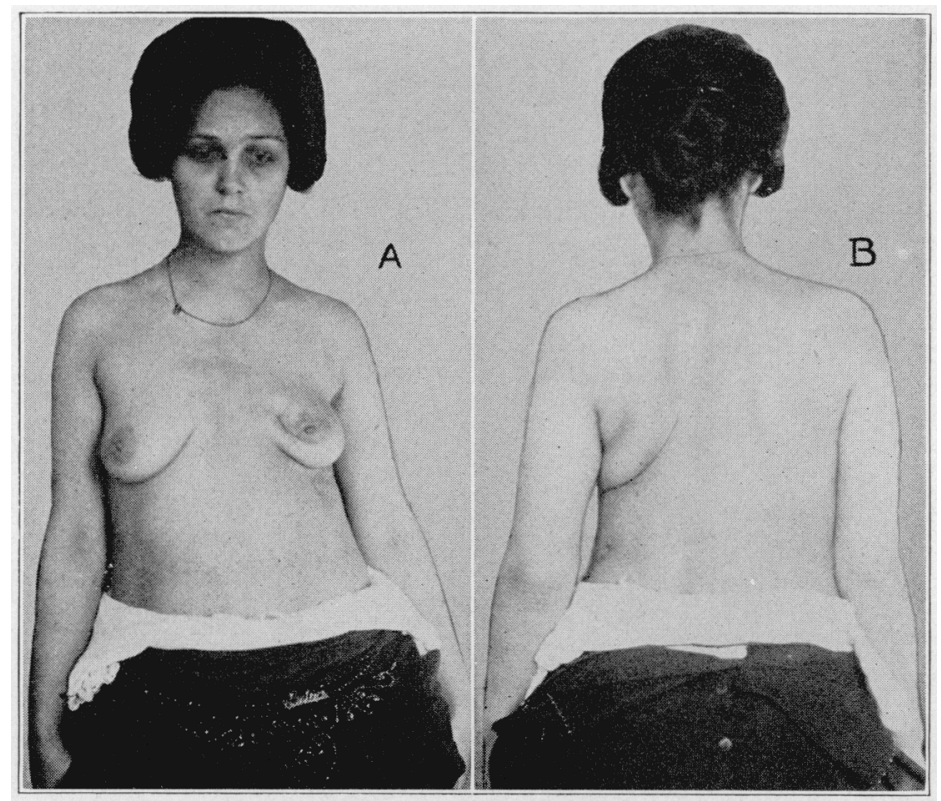

Fig. 6.-A, anterior view of patient six weeks after operation; $B$, posterior view six weeks after opcration.

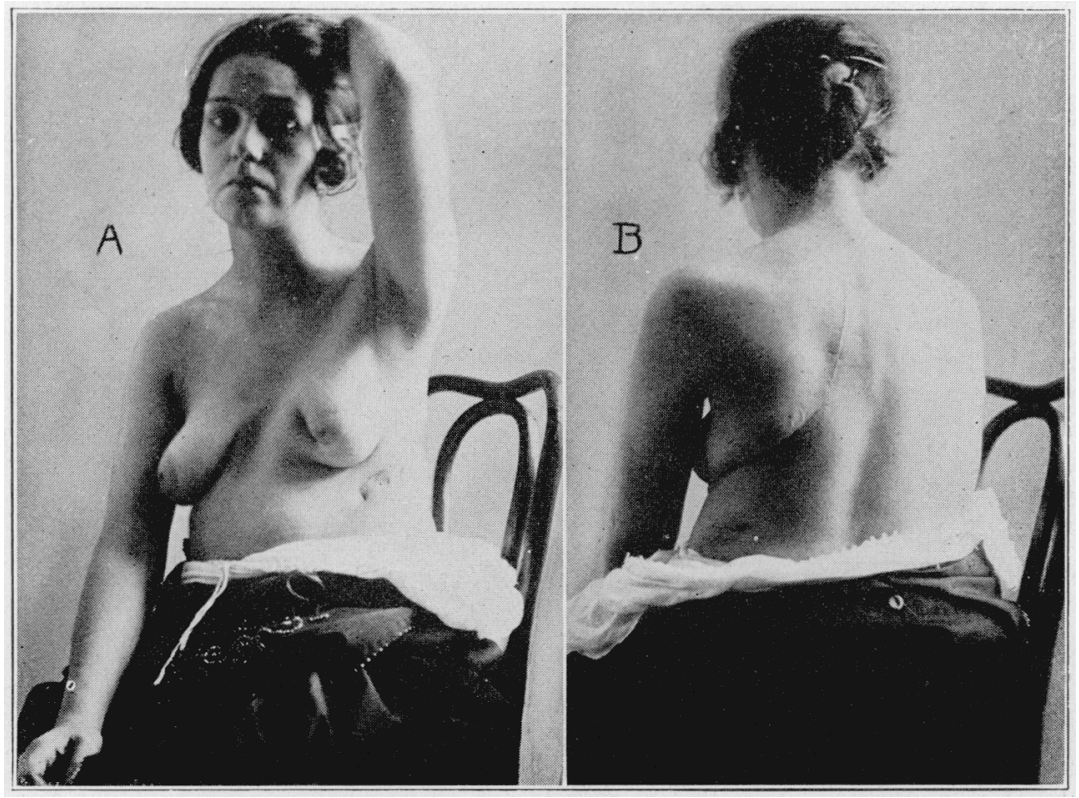

Fig. 7.-A, patient, six weeks after operation, showing lack of interference with function of the arm; $B$, posterolateral view showing operation wound. 
able to get about without undue fatigue. She weighed 107 pounds $(48.5 \mathrm{~kg}$.) and breath sounds of a blowing character were heard through the chest, except toward the midline posteriorly.

The left shoulder was practically on a level with the right. The deviation of the spinous processes to the right was less than $2 \mathrm{~cm}$.

Since leaving the hospital, the patient has been repeatedly examined. There has been a continuous improvement in her general health, although late in January she had an attack of diphtheria. There is now, May 30, 1921, moderately good expansion of the left side of the chest, and, although it is obvious on examination that the left half of the chest is smaller than the right, when she is wearing her clothing the deformity is not noticeable. She is at present engaged at work as a ward maid in the hospital. She weighs 129 pounds $(58.4 \mathrm{~kg}$.).

\section{COMMENT}

Although in no other case have I encountered such a marked degree of scoliosis, in all cases in which chronic infected open pneumothorax has persisted for six months or longer, there is marked lateral curvature of the spine. Ten cases of this kind have been observed in which correction of the deformity has followed extensive thoracotomy. In one patient, who is still under observation, decortication of the lung did not prove successful in inducing obliteration of the cavity, and an extensive resection of the chest wall was performed. Resection of the posterior segments of all the ribs, from the second to the tenth, inclusive, was performed. This interference has been followed not only by correction of the curvature of the spine toward the healthy side, but also by a moderate scoliosis projecting toward the diseased side, as demonstrated by roentgenograms. Apparently, in this case, the complete removal of pressure of the ribs upon the spine has resulted in deviation of the spine to the unsupported side.

\section{CONCLUSION}

Chronic infected open pneumothorax if long continued is followed by scoliosis. Curvature of the spine is toward the healthy side of the chest, and is consequent upon pressure exerted upon the spine by the contracting ribs on the diseased side.

Correction of the deformity may be accomplished by means of an extensive thoracotomy with removal and division of several ribs. 